\title{
Research and Realization of Poc Group List Management Server
}

\author{
Dawei Ma, Binde Luo \\ Mobilization Center Of Civil-military Connection \\ Terminal Equipment In $3 \mathrm{G}$ \\ Chongqing Communication Institute \\ Chongqing China, 400035
}

\author{
Fengbin Zhang, Chen Sheng \\ Mobilization Center Of Civil-military Connection \\ Terminal Equipment In 3G \\ Chongqing Communication Institute \\ Chongqing China, 400035
}

\begin{abstract}
PoC is a part of the IP Multimedia Subsystem service, and provides one to one and group call through a halfduplex VoIP technology. The Group List Management Server (GLMS) in PoC is the auxiliary functional entity to provide services for the PoC contact list management, group list management, etc group business capabilities. In paper the GLMS as the research object is analyzed of the PoC service needs. Finally, a PoC GLMS is designed and realized.
\end{abstract}

Keywords--PoC, Group list managemen, Pre-arranged PoC Group User

\section{INTRODUCTION}

PUSH-TO-TALK over Cellular (PoC) [1] is a "walkietalkie" service designed for mobile networks. In this service, several predefined $\mathrm{PoC}$ group members participate in one $\mathrm{PoC}$ session. Since the PoC session is half-duplex, only one PoC member speaks at a time, and the others listen. When a PoC member attempts to speak, he presses the button of his mobile terminal to apply the permission. This mobile terminal with push-to-talk function is called the PoC client.

The Group List Management Server (GLMS) is used as a lonely service in Open Mobile Alliance. As an Enabler it can provide the list management and storage for PoC, Instant Messaging (IM), and Presence etc. XML configuration Access Protocol (XCAP) and session Initiation Protocol (SIP) is used by GLMS and Client. GLMS is a service function, and can provide personal information management, group information management and special service information management.

The remainder of the paper is organized as follows. In Section 2, the PoC networking program is discussed. In section 3, the group list management rule is proposed, and its character is introduced. In section 4, the GLMS is realized by eclipse 6.0 and the Android SDK 2.0. Finally, a brief conclusion is made in section 5 .

\section{The Research Of The Networking Program IN POC}

\section{A. PoC Network Structure}

In the Fig 1, there are many logic entities which are briefly introduced as follows:

(1) The side of the UE: Its main part is a PoC Client which is used for the call processing, the media processing and so on. XDMC is used for the group management function. Management operation can be realized in mobile phone. The presence service is achieved by Presence Source and Watcher. Equipment management function is realized by DM Client.

(2) Network Management: It is consisted of the Aggregation, Proxy, PoC XDMS, Shared XDMS and XDM Administration, which provides the function of adding, modifying, deleting, empowering and control for $\mathrm{PoC}$ group information.

(3) Presence Server: It is used to receive, save, and dispense the messages of presence. Its function module is consisted of Presence Server, Presence XDMS Resource List Server and RLS XDMS. There are three presence states "On line", "off line" and "ISB".

(4) SIP/IP Core: SIP Proxy is another access node of the PoC service platform. At this time, PoC client uses SIP protocol to visit the $\mathrm{PoC}$ service platform. Its functions include the parsing of SIP address, the maintenance of registration state about SIP layer, the presence state of the user, the authentication and authorization of users; and the billing information.

\section{B. GLMS in PoC System}

The GLMS in PoC system is the accessibility entities of the PoC server. It is not directly involved in the PoC Server to the PoC session establishment and control, which is used by $\mathrm{PoC}$ users to store their contact information list, group list function and access control policy on the server. The GLMS in PoC system provides the group access capability for the PoC Server, and supports all specific functions in PoC. Figure 3 shows the logical structure of the OMA PoC specification in $\mathrm{PoC}$ system. Among them, The XML Document Management achieves GLMS function [2]. 


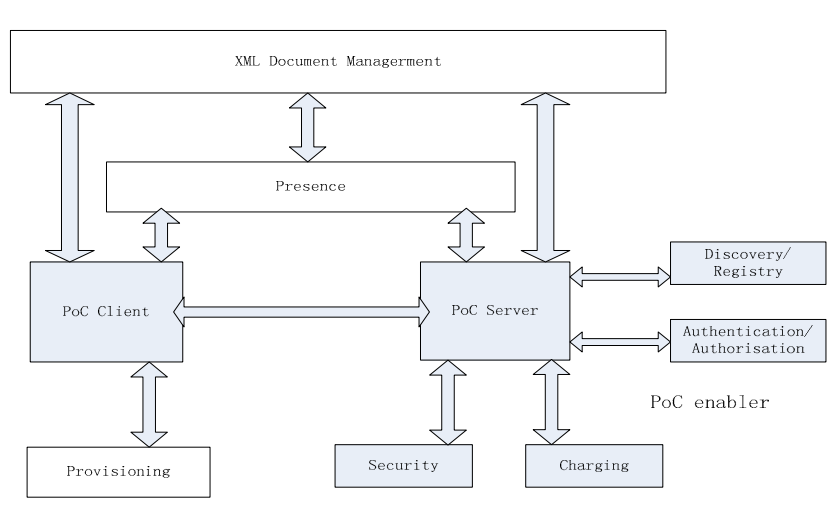

Figure 2 The logic architecture of PoC system

GLMS provides the following features for the PoC:

(1) Provide a list of management operations. For example, the creating, modifying, reading and deleting of the group list;

(2) Provide a storage of group list;

(3) Access control policy management [3].

\section{Group List MANAGEMENT}

The group list management is defined by OMA PoC $\mathrm{XDM}$, and its aims at achieving the creation, deletion, updating, and query for group list and group member [4]. In $\mathrm{PoC}$ session, the group is created by an owner, and the other users are invited to join the group. The members of the group can look over the information of the group. Usually, only the group owner who creates the group can update and delete the group information. The group information includes the group name, PoC special service information, the visibility and the permission right. The group owner can invite others to join the group, and delete someone from the group. The group members can modify themselves attributes and exit the group in permission.

The partly XCAP rules is described by OMA PoC XDM as follows :

(1) AUID is org.openmobilealiance PoC-groups;

(2) The style of the MIME is application/vnd.oma. poc.groups $+\mathrm{xml}$;

(3) Namespace is um:oma:: xml:PoC:list-service;

(4) Users tree stored in the users list of each group is only one list-service elements;

(5) Group list should use the form of SIP UIR. And, the SIP UIR in group should be the only.

Group inquiry function is defined by the following mechanism: in the index document of the global tree which can have more than one list-service elements. All users group list is stored in the index documents. This PoC Server can be used as a XDMC to send XCAP request, and get the contents of the group by the group label. That is, if only the users tree of the document is changed (such as create, delete and update), the index documents of the global tree should be changed. So, PoC XDMS should avoid the ordinary users to change the index document of the global tree.

\section{SimUlation}

The PoC service management system is developed by Myeclipse 8.0 and Mysql database in Linux. Its main functions include: the adding, deleting, modifying and updating users, and the adding temporary users.

\section{A. Database Design}

TABLE I. ADMINISTRATOR LOGIN

\begin{tabular}{|c|c|c|}
\hline Field Name & Field Type & Meaning \\
\hline ID & integer & Key Word \\
\hline Username & integer & Username \\
\hline Password & integer & Login password \\
\hline
\end{tabular}

Mysql database needs three forms to store data as follows:

(1) Login: It stores login information of administrator user;

(2) User: It stores user information, such as user number, username, user level, and group number;

(3) Groupuser: It stores the pre-defined user group number and the temporary number group which is generally defined as no more than 10 users.

TABLE II. USERS LIST

\begin{tabular}{|c|c|c|}
\hline Field Name & Field Type & Meaning \\
\hline ID & integer & Key Word \\
\hline Username & integer & Communications User Name \\
\hline Usernumber & integer & Communications User number \\
\hline Userlevel & integer & Communications user priority \\
\hline Groupnumber & integer & $\begin{array}{c}\text { Communications User Group } \\
\text { Number }\end{array}$ \\
\hline
\end{tabular}

TABLE III. TEMPORARY GROUP USERS LIST

\begin{tabular}{|c|c|c|}
\hline Field Name & Field Type & Meaning \\
\hline ID & Integer & Key Word \\
\hline Groupnumber & Integer & Temporary group number \\
\hline User1 & Integer & Temporary group members \\
\hline$\ldots$ & $\ldots$ & $\ldots$ \\
\hline User10 & Integer & Temporary group members \\
\hline
\end{tabular}

\section{B. WEB Development}

Myeclipse is used to develop WEB which connects to the database. The JSP language is used to add, delete, change, check users, and modify black list, white list

The part codes connected to the database is as follows:

$<\%$ page language="java" import="java.util.*" pageEncoding="UTF-8"\%>

$<\% @$ page contentType="text/html; charset=gb2312" language="java" import="java.sql.*" \%>

$<\%$ ResultSet rs;

Connection conn;

String username=request.getParameter("userName");

String password=request.getParameter("passwd");

String driverName="com.mysql.jdbc.Driver"; 
Class.forName(driverName).newInstance();

String url="jdbc:mysql://localhost:3306/mysql"; conn.close();

$$
\ldots . . \%>
$$

The WEB is used to achieve the group list management services, and it is appropriate to visit the group list management server simultaneity by the terminal and the server. Figure 3 is the development interface for the server management.

\section{Function Realization}

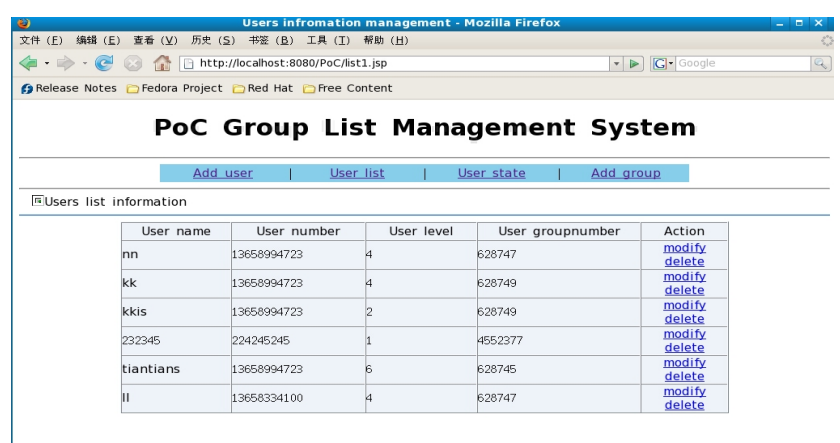

Figure 3. The WEB of the server management

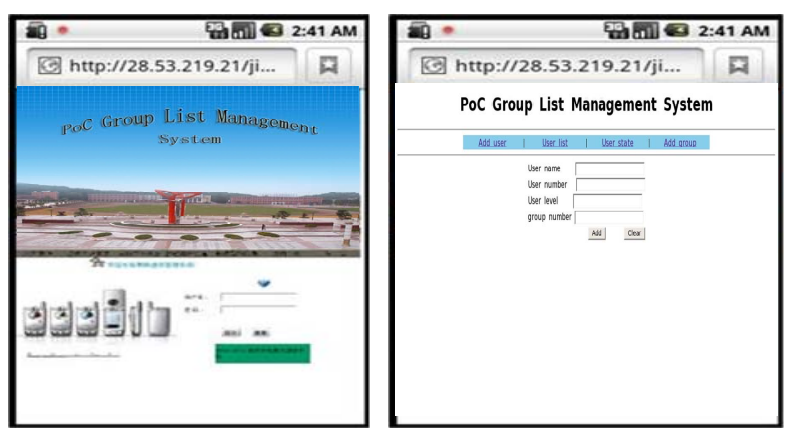

Figure 4. Android phone access the GLMS
WEB GLMS is to access by eclipse 6.0 and the Android SDK 2.0. The PoC was implemented by adding, deleting, modifying user group list information, and adding, deleting temporary groups of users. Figure 4 is a screenshot through Android phones to access GLMS. From this we achieve group list management through GLMS. It is used to achieve the PoC service [5].

\section{CONCLUSIONS}

In this paper, the GLMS is analyzed. We design a GLMS by WEB and database. The job will work for PoC service in next step. On the one hand, PoC service can open up new markets, and does not affect the original voice services. On the other hand, it can increase data revenue. The operator's Average Revenue Per User (ARPU) is improved effectively. GLMS as PoC system support subsystems will play its due role.

\section{REFERENCES}

[1] OMA, Push to talk over cellular (PoC) - architecture, OMA-ADPoCV2.0 1-20080226-C Candidate Version 2.0[S], 2008, 9.

[2] Binde Luo, Zhiyong Shi, Dawei Ma, YIN Shi-ping. Design and Capacity Analysis of PoC Service Based on TD-SCDMA Network, The 6th International Conference on Wireless Communications, Networking and Mobile Computing[C], 2010.

[3] GPP TS 24.228, v.5.3.0, Signaling delay in 3GPP. Fremantle WA: Sixth International Symposium Communications Interworking of IFIP-Interworking[C], 2002, PP: 211-222.

[4] LUO Bin-de, SHI Zhi-yong, Ma DA-wei. Study on Group Call Based on TD Network (in Chinese), Communications Technology[J].2010. 6, PP:163-165.

[5] Vehmas A. "Service adoption for push over cellular"[EB/OL], 2004, web page: www.tml.hut.fi/Opinnot/T-109.551/2004/reports/poc.pdf

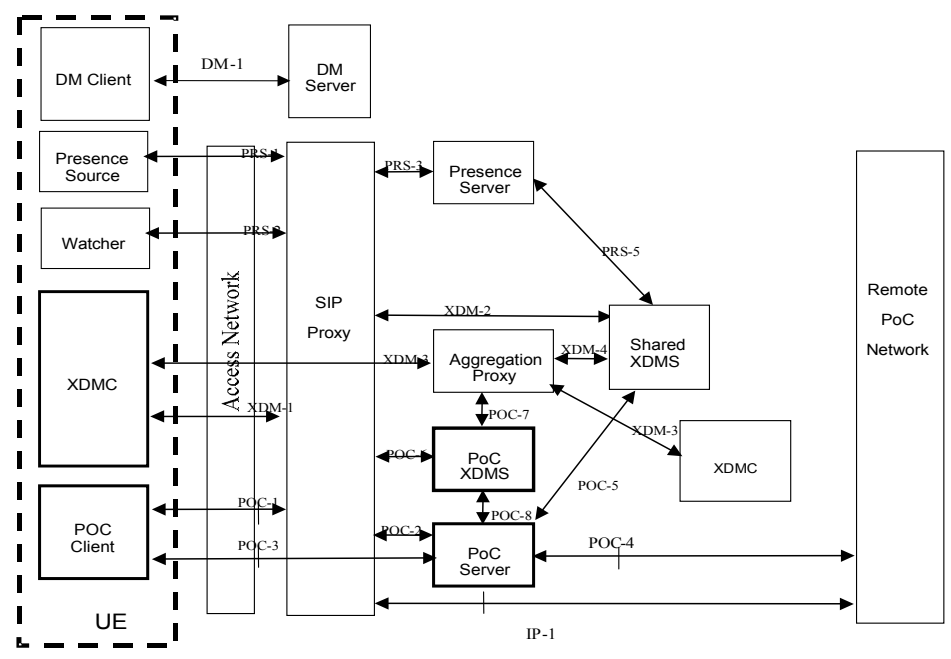

Figure 1. PoC architecture 